\title{
Ternary positive quadratic forms that represent all odd positive integers
}

\author{
by
}

\author{
IRVING KAPLANSKY (Berkeley, Cal.)
}

1. Introduction. The motivation for this work comes from the fact that $x^{2}+y^{2}+2 z^{2}$ represents all odd positive integers. This seems to me to be of special interest among results on ternary forms; for instance, in 1785 Legendre remarked that it could be regarded as a strengthening of Lagrange's four squares theorem [2, p. 282]. I therefore thought it would be a useful step forward to determine all the positive ternary forms that share this property.

According to my search of the literature, this question has not been previously raised. There is, however, a similar project of finding all positive quaternary forms that represent all positive integers; this started with Ramanujan [6] and, after many intervening papers, culminated with Willerding [7].

In this paper I report partial progress on the problem. There are at most 23 such forms - 19 for sure and 4 plausible candidates; they are listed at the end of the paper. The 4 candidates do represent all odd numbers up to 16383 $\left(=2^{14}-1\right)$. I am greatly indebted to Noam Elkies who kindly programmed and ran off the computation.

For 18 of the 19 forms more is true: they are regular, i.e., each represents all numbers (even as well as odd) represented by its genus. (See [4] for background on regular forms.) For 15 of the 18 this is true simply because each is alone in its genus. I believe that regularity of the 3 other forms is proved here for the first time; the proofs appear in Section 3. An ad hoc proof that the 19th form represents all odd positive integers is presented in Section 4.

In addition I shall mention three forms that I am calling "near misses":

$$
\begin{gathered}
x^{2}+y^{2}+9 z^{2}+x y+x z, \quad x^{2}+2 y^{2}+5 z^{2}+x z+2 y z, \quad \text { and } \\
x^{2}+3 y^{2}+5 z^{2}+x y+y z .
\end{gathered}
$$

Each seems to represent all odd positive integers with exactly one exception. 
More precisely, up to 16383 they represent all odd positive integers except 5,13 , and 17 , respectively.

Remarks. (a) For the computations on the near misses I am also indebted to Noam Elkies. (b) I was not looking for near misses and so there may be others. (c) For positive diagonal quaternary forms Halmos [3] (with a final touch added by Pall [5]) found all near misses, relative to all positive integers. (d) I have additional information on the last of the near misses (call it $k$ ) which I mention without proof. First, $k$ represents all odd positive integers congruent to 0 or $1(\bmod 3)$. Second, let $A$ be an odd positive integer congruent to $2(\bmod 3)$. Then $k$ represents $A$ if and only if $A$ can be written $x^{2}+2 y^{2}+3 z^{2}$ with $z$ prime to 3 . (Of course, both statements fail for $A=17$ and otherwise hold up to 16383.)

2. Modus Operandi. The investigation involved a fair amount of hand computation which, I feel, is unsuitable for public scrutiny. However, I shall outline the procedure. To diminish the possibility of error I did the work twice, using different methods. In the first method the assumption that the form represents the first few odd numbers produced a reasonably short list of eligible discriminants. In the second method an a priori upper bound for the discriminant was found and all forms up to this bound were examined. I am indebted to John Hsia who sent me a copy of the relevant portion of the massive table [1].

First the forms were tested for representing all odd positive integers locally; this is an easy thing to check. If a form passed this test and was alone in its genus it was forthwith mounted as a specimen. It turned out that 8 forms were left. As noted above, I proved that 3 are regular and gave a special argument for a fourth. The remaining 4 resisted my attacks and remain in limbo. I hope that their fate will be settled some day.

3. Three proofs of regularity. The proofs follow a single plan. In each case there is one other form in the genus; I write $f$ for the target form and $g$ for its genus mate. Let $A$ be an integer represented by the genus. If $g$ does not represent $A$, then $f$ does. So I may assume that $g$ represents $A$.

I. $f=x^{2}+3 y^{2}+3 z^{2}+x y+3 y z$ (number 13 on the list), $g=x^{2}+x y+y^{2}$ $+8 z^{2}$. It is known that the binary forms $x^{2}+x y+y^{2}$ and $x^{2}+3 y^{2}$ represent exactly the same numbers. Hence we can replace $g$ by $x^{2}+3 y^{2}+8 z^{2}$. Thus there exist $u, v, w$ with $A=u^{2}+3 v^{2}+8 w^{2}$. Set $x=u-w, y=2 w$, $z=v-w$. Then $f(x, y, z)=A$; I leave this short computation to the reader.

II. $f=x^{2}+3 y^{2}+3 z^{2}+x y+y z$ (number 15 on the list), $g=x^{2}+y^{2}+$ $11 z^{2}+x y+x z$. If $g(u, v, w)=A$ we have

$$
12 A=(3 u+2 w)^{2}+3(u+2 v)^{2}+128 w^{2},
$$


another computation that I omit. The following is known: if $p^{2}+3 q^{2}$ is a multiple of 4 then $\left(p^{2}+3 q^{2}\right) / 4$ can also be written as $r^{2}+3 s^{2}$. On applying this to (1) and dividing by 4 we get

$$
3 A=r^{2}+3 s^{2}+32 w^{2} .
$$

Now $r^{2}+32 w^{2}$ is divisible by 3 and hence so is $r^{2}-w^{2}$. By changing the sign of $w$, if necessary, we arrange that $r-w$ is divisible by 3 . Set $x=s-w, y=2 w, z=(r-w) / 3$. Then $f(x, y, z)=A$. This time I give a little detail:

$$
\begin{aligned}
f(x, y, z)= & (s-w)^{2}+3(2 w)^{2}+3[(r-w) / 3]^{2} \\
& +2 w(s-w)+2 w(r-w) / 3 \\
3 f(x, y, z)= & 3\left(s^{2}-2 s w+w^{2}\right)+36 w^{2}+r^{2}-2 r w+w^{2} \\
& +6\left(s w-w^{2}\right)+2 r w-2 w^{2} \\
= & r^{2}+3 s^{2}+32 w^{2}
\end{aligned}
$$

which is $3 A$, by $(2)$.

III. $f=x^{2}+3 y^{2}+5 z^{2}+x z+y z$ (number 19 on the list), $g=x^{2}+y^{2}+$ $19 z^{2}+x y+x z$. The details are nearly the same as in II and I shall be brief. With $g(u, v, w)=A$ we have

$$
12 A=3(u+2 v)^{2}+(3 u+2 w)^{2}+224 w^{2}, \quad 3 A=r^{2}+3 s^{2}+56 w^{2} .
$$

With $w$ changed, if necessary, to make $r-w$ divisible by 3 we set $x=s-w$, $y=(r-w) / 3, z=2 w$. Then

$$
\begin{aligned}
3 f(x, y, z) & =3(s-w)^{2}+(r-w)^{2}+15(2 w)^{2}+6 w(s-w)+2 w(r-w) \\
& =r^{2}+3 s^{2}+56 w^{2}=3 A .
\end{aligned}
$$

4. Form number 18. $h=x^{2}+3 y^{2}+5 z^{2}+x y+x z-y z$. In the following proof no use is made of the other form in the genus of $h$.

First we present a lemma. This lemma is surely well known; for lack of a reference we include a proof.

LEMMA. If a nonzero integer $r$ is divisible by 5 and expressible as a sum of two squares then $r$ can be written $p^{2}+q^{2}$ with both $p$ and $q$ prime to 5 .

Proof. The integer $s=r / 5$ is also a sum of two squares. We claim that $s$ can be written $s=t^{2}+u^{2}$ with at least one of $t, u$ prime to 5. For: if $s$ is prime to 5 , this is automatic, and if $s$ is divisible by 5 , we can arrange to have both $t$ and $u$ prime to 5 by induction. Suppose for definiteness that $u$ is prime to 5 . Change the sign of $u$, if necessary, so that $t \not \equiv 2 u(\bmod 5)$. We have

$$
(t-2 u)^{2}+(2 t+u)^{2}=5\left(t^{2}+u^{2}\right)=5 s=r .
$$

Note that $t-2 u$ is prime to 5 . It follows that $2 t+u$ is also prime to 5 . 
We turn our attention to $h$. The first step is going to be a switch to an equivalent form; the version at hand is the canonical form as given in [1] but it will be more convenient to use a version with one of the cross product terms vanishing. We set $x=X+Y, y=Y, z=Z$. The form becomes

$(X+Y)^{2}+3 Y^{2}+5 Z^{2}+(X+Y) Y+X Z=X^{2}+5 Y^{2}+5 Z^{2}+3 X Y+X Z$.

We change notation and write $h=x^{2}+5 y^{2}+5 z^{2}+3 x y+x z$.

Let $A$ be an odd positive integer. Our task is to prove that $h$ represents $A$. We can assume that $A$ is not divisible by 25 , for if it is, $h$ represents $A / 25$ by induction and we need only multiply the variables by 5 . (Of course, if it helped, we could assume $A$ to be square-free.) It is known that $2 A$ is representable as a sum $c^{2}+d^{2}+e^{2}$ of three squares. They cannot all be divisible by 5 ; say $c$ is prime to 5 . We cannot have $d=e=0$, for then $2 A$ would be a square, whereas it is twice an odd number. The lemma is applicable to $r=10\left(d^{2}+e^{2}\right)$ and we write $r=a^{2}+b^{2}$ with $a$ and $b$ prime to 5 . We have

$$
20 A=a^{2}+b^{2}+10 c^{2}
$$

with $a, b$, and $c$ all prime to 5 . The next claim is that $a, b$, and $c$ all have the same parity. For if $c$ is odd, $a^{2}+b^{2} \equiv 2(\bmod 4)$ by $(3)$ and $a$ and $b$ have to be odd; if $c$ is even, $a^{2}+b^{2} \equiv 0(\bmod 4)$ and $a$ and $b$ have to be even. Note next that each of $a^{2}, b^{2}$, and $c^{2}$ is congruent to $\pm 1 \bmod 5$. Furthermore, since $a^{2}+b^{2} \equiv 0(\bmod 5)$, one of $a^{2}, b^{2}$ is congruent to 1 and the other to -1 . Interchange $a$ and $b$, if necessary, so that $a^{2} \equiv c^{2}, b^{2} \equiv-c^{2}(\bmod 5)$. Change the sign of $a$, if necessary, so as to achieve $a \equiv c(\bmod 5)$. We have $b^{2}-9 c^{2} \equiv 0(\bmod 5)$. Change the sign of $b$, if necessary, so as to achieve $b \equiv 3 c(\bmod 5)$. We now have $a-c$ and $b-3 c$ both divisible by 10 . We are at last ready to assign the values of $x, y$, and $z: x=c, y=(b-3 c) / 10$, $z=(a-c) / 10$. This final computation is going to be left to the reader: we have $20 h(x, y, z)=$ the right side of $(3)$.

5. The list. It takes only a few seconds to discuss diagonal forms. Three forms emerge: the first three on the list. It takes just a little longer to handle the more general case of forms with even cross product coefficients: the next two forms emerge. Serious business began when I attacked forms with at least one odd cross product coefficient. The survivors are listed in order of increasing discriminant; the discriminants are the numbers in parentheses. I am following the normalization of the discriminant used in [1].

1. $x^{2}+y^{2}+2 z^{2}$

2. $x^{2}+2 y^{2}+3 z^{2}$

3. $x^{2}+2 y^{2}+4 z^{2}$ 
4. $x^{2}+3 y^{2}+2 y z+3 z^{2}$

5. $x^{2}+3 y^{2}+2 y z+5 z^{2}$

6. (2) $x^{2}+y^{2}+z^{2}+x y+x z$

7. (6) $x^{2}+x y+y^{2}+2 z^{2}$

8. (8) $x^{2}+y^{2}+3 z^{2}+x y+x z$

9. (10) $x^{2}+y^{2}+3 z^{2}+x z+y z$

10. (14) $x^{2}+y^{2}+5 z^{2}+x y+x z$

11. (18) $x^{2}+2 y^{2}+3 z^{2}+x z+2 y z$

12. $(22) x^{2}+2 y^{2}+3 z^{2}+x z$

13. (24) $x^{2}+3 y^{2}+3 z^{2}+x y+3 y z$

14. (30) $x^{2}+3 y^{2}+3 z^{2}+x y+x z$

15. (32) $x^{2}+3 y^{2}+3 z^{2}+x y+y z$

16. (40) $x^{2}+3 y^{2}+4 z^{2}+x y+2 y z$

17. (46) $x^{2}+3 y^{2}+5 z^{2}+x y+3 y z$

18. (50) $x^{2}+3 y^{2}+5 z^{2}+x y+x z-y z$

19. $(56) x^{2}+3 y^{2}+5 z^{2}+x z+y z$

Candidates

20. (38) $x^{2}+2 y^{2}+5 z^{2}+x z$

21. (62) $x^{2}+3 y^{2}+6 z^{2}+x y+2 y z$

22. $(72) x^{2}+3 y^{2}+11 z^{2}+x y+7 y z$

23. $(74) x^{2}+3 y^{2}+7 z^{2}+x y+x z$

\section{References}

[1] H. Brandt und O. Intrau, Tabelle reduzierten positiver ternärer quadratischer Formen, Abh. Sächs. Akad. Wiss. Math.-Nat. Kl. 45 (1958), no. 4, MR 21, 11493.

[2] L. E. Dickson, History of the Theory of Numbers, Vol. II, Chelsea reprint, 1952.

[3] P. R. Halmos, Note on almost-universal forms, Bull. Amer. Math. Soc. 44 (1938), 141-144.

[4] J. S. Hsia, Regular positive ternary quadratic forms, Mathematika 28 (1981), 231238.

[5] G. Pall, An almost universal form, Bull. Amer. Math. Soc. 46 (1940), 291

[6] S. Ramanujan, On the expression of a number in the form $a x^{2}+b y^{2}+c z^{2}+d u^{2}$, Proc. Cambridge Philos. Soc. 19 (1917), 11-21; Collected Works, 169-178. 
[7] M. F. Willerding, Determination of all classes of positive quaternary quadratic forms which represent all positive integers, Bull. Amer. Math. Soc. 54 (1948), 334337.

MATHEMATICAL SCIENCES RESEARCH INSTITUTE

1000 CENTENNIAL DRIVE

BERKELEY, CALIFORNIA 94720-5070

U.S.A.

Received on 19.4.1994

and in revised form on 15.9.1994 